\title{
Importance of bulk states for the electronic structure of semiconductor surfaces: implications for finite slabs
}

\author{
Keisuke Sagisaka ${ }^{1,2}$, Jun Nara ${ }^{3}$, David Bowler ${ }^{4}$ \\ ${ }^{1}$ Research Center for Advanced Measurement and Characterization, National \\ Institute for Materials Science \\ 1-2-1 Sengen, Tsukuba, Ibaraki, 305-0047 Japan \\ ${ }^{2}$ London Centre for Nanotechnology, 17-19 Gordon St, London, WC1H 0AH, U.K. \\ ${ }^{3}$ International Centre for Materials Nanoarchitectonics (MANA), National Institute \\ for Materials Science \\ 1-1 Namiki, Tsukuba, Ibaraki, 305-0044 Japan \\ ${ }^{4}$ Thomas Young Centre and Department of Physics \& Astronomy, University College \\ London \\ Gower St, London, WC1E 6BT, U.K \\ E-mail: SAGISAKA.Keisuke@nims.go.jp, david.bowler@ucl.ac.uk
}

\begin{abstract}
We investigate the influence of slab thickness on the electronic structure of the $\operatorname{Si}(100)-p(2 \times 2)$ surface in density functional theory (DFT) calculations, considering both density of states and band structure. Our calculations, with slab thicknesses of up to 78 atomic layers, reveal that the slab thickness profoundly affects the surface band structure, particularly the dangling bond states of the silicon dimers near the Fermi level. We find that, to precisely reproduce the surface bands, the slab thickness needs to be large enough to completely converge the bulk bands in the slab. In case of the $\operatorname{Si}(100)$ surface, the dispersion features of the surface bands, such as the band shape and width, converge when the slab thickness is larger than 30 layers. Complete convergence of both the surface and bulk bands in the slab is only achieved when the slab thickness is greater than 60 layers.
\end{abstract}

Keywords: density functional theory, band structure, semiconductor surface, silicon, slab thickness 


\section{Introduction}

Semiconductor surfaces are of enormous importance both industrially, as the basis of the microelectronics industry, and scientifically, where they form the basis for studies of diffusion, growth and electronic structure. Moreover, recently a number of proposals for quantum information implementations based on isolated states in or near semiconductor surfaces have emerged [1]. In light of this, it is vital that our understanding of their atomic and electronic structure is as accurate and detailed as possible. We demonstrate in this paper that both the gap and the character of the valence band depend strongly on the thickness of slabs used to model the system, and find that the slabs used in most calculations are much too small to describe the electronic structure of $\mathrm{Si}(100)$, the most common surface in industry.

Density functional theory (DFT) has become the standard approach to model the atomic and electronic structures of semiconductor surfaces. Although it is known to underestimate the band gap of solids, DFT generally reproduces the density of states (DOS) and band structure accurately. A surface is usually modeled by using a supercell consisting of a slab with two surfaces and a vacuum region. Since this approach gives a non-periodic system perpendicular to the surface (z direction), the accuracy of the resulting electronic structure will depend on the slab thickness. In case of the Ge(100) surface, which bears a very similar structure to $\mathrm{Si}(100)$, the influence of slab thickness on the top of the valence band has been discussed in detail [2, 3, 4, 5]. Some other physical properties, such as surface electronic structure [6], dielectric function [7], chemical reactivity [5], and transport properties [8], sensitively vary with cell size and slab thickness. Since recent improvements to experimental techniques allow measurements to be made with high energy resolution $(\sim$ meV), slab thickness is likely to become a key parameter in determining energy precision in DFT modeling.

However, it is still unclear how thick a slab is needed to reliably produce the electronic structure of a semiconductor surface; one study 5 suggested that slab thickness was unimportant, while another study of the basic $(2 \times 1)$ reconstruction [9] suggested, without giving detailed analysis, that at least 30 layers are required. Recently, we found that previously reported band structures of $\mathrm{Si}(100)$ calculated with DFT [10, 11, 12] do not compare well with data obtained by the state-of-the-art angleresolved photo-emission spectroscope (ARPES) [13]. In addressing this problem, we found that surprisingly thick slabs were required to obtain agreement with the ARPES data. In this paper, we report the critical slab thicknesses in the DFT calculation required to reliably produce the electronic structure of the $\mathrm{Si}(100)$ surface in terms of DOS and band structure.

\section{Computational method}

DFT calculations were carried out using the VASP code with a plane wave basis set [14, 15. We employed PAW and the GGA PBE exchange correlation functional 
[16]. The $\operatorname{Si}(100)-p(2 \times 2)$ surface was modeled using supercells consisting of a Si slab and vacuum layer of approximately $15 \AA$ thick (this vacuum thickness was carefully converged). The bottom layer was terminated with hydrogen atoms and the bottom layer and hydrogen layer were fixed; as the system is not polar, and the bottom surface is inert, no dipole corrections are required. For total energies and DOS, the tetrahedron method was applied [17]. Full details are given below.

Parameters used We tested the influence of slab thickness between 6 and 78 atomic layers (L) on the electronic structure of the slab, in particular the dangling bond (DB) states. To ensure full convergence with respect to the basis set, we employed a plane wave cutoff energy of $312.5 \mathrm{eV}$ throughout this study. Structural relaxation was done with Brillouin zone (BZ) sampling of $8 \times 8 \times 1$ Monkhorst-Pack k-point mesh until the forces on each atom reached to below $0.02 \mathrm{eV} / \AA$. The relaxed lattice constant was $a_{0}=$ $5.466 \AA$, which is slightly larger than experimental value $(5.43 \AA)$. For the total energies and DOS plots, the BZ sampling was increased to $22 \times 22 \times 1$.

Surface energies Surface energies were calculated using both a reconstructed slab and a fully hydrogen terminated slab. The total energies were calculated for the $\mathrm{p}(2 \times 2)$ slabs (the top was dimerised, the bottom was terminated with hydrogen) and hydrogen terminated slabs (both surfaces were terminated with hydrogen, using the same structure as the slab base) for thicknesses of $6 \mathrm{~L}, 14 \mathrm{~L}, 22 \mathrm{~L}, 30 \mathrm{~L}, 38 \mathrm{~L}, 62 \mathrm{~L}$ and $78 \mathrm{~L}$. All simulation cell sizes were constant $(2 \times 2 \times 78 \mathrm{~L})$. As the slab thickness was varied, so the amount of vacuum space also varied. Each $\mathrm{p}(2 \times 2)$ slab was relaxed with a $8 \times 8 \times 1 \mathrm{k}$-point sampling, then the total energy was calculated with a $22 \times 22 \times 1 \mathrm{k}$-point sampling, with the tetrahedron method. By contrast, each hydrogen terminated slab was unrelaxed. The total energy was calculated with a $22 \times 22 \times 1 \mathrm{k}$-point sampling, again using the tetrahedron method. For all these calculations, the plane wave cutoff was increased to $600 \mathrm{eV}$.

To find a bulk silicon atomic energy, the total energy was calculated for a periodic $2 \times 2 \times 4$ cell (16 Si atoms, bulk calculation), which was then divided by 16 to give the energy of a Si atom.

Then, the surface energy was found by subtract the energy for hydrogen termination (for one surface) and the energy of the bulk silicon atoms from the energy for the $\mathrm{p}(2 \times 2)$ cell, divided by 2 to give $\mathrm{eV} /$ dimer.

Band Structure For band structures and densities of states, we performed a static calculation with $22 \times 22 \times 1 \mathrm{k}$-point sampling to produce a charge density. $(4 \times 4 \times 1$ for the hybrid calculation)

To find a consistent energy zero for comparison between different slab thicknesses, all eigen values were shifted relative to the potential at the Si core, averaged over the first five layers below the surface. The top of the valence band of the $2 \times 1$ symmetric 38L in Fig. 4(d) was arbitrarily chosen as the zero for all figures. All bands in Fig. 4 
Table 1. Dimer bond length, dimer buckling angle, and surface energy obtained for the $\operatorname{Si}(100) p(2 \times 2)$ surface with varying slab thicknesses. For surface energy calculations, we used a constant cell size $\left(7.731 \times 7.731 \times 121.914 \AA^{3}\right)$ with different slab thicknesses and increased the cutoff energy up to $600 \mathrm{eV}$.

\begin{tabular}{llll}
\hline Slab thickness (layers) & Dimer bond length $(\AA)$ & Buckling angle $\left(^{\circ}\right)$ & Surface energy $(\mathrm{eV} /$ dimer $)$ \\
\hline 6 & 2.360 & 19.0 & 2.424 \\
14 & 2.357 & 19.1 & 2.446 \\
22 & 2.355 & 18.9 & 2.331 \\
30 & 2.355 & 19.1 & 2.331 \\
38 & 2.357 & 19.1 & 2.332 \\
62 & 2.357 & 19.1 & 2.333 \\
78 & 2.357 & 19.0 & 2.332 \\
\hline
\end{tabular}

in the main text are drawn with respect to a consistent energy zero, the potential value at the Si cores.

Bulk band gap for a slab The bulk band gap of a slab was found by considering the projected DOS of Si atoms in the middle layer of the slab. We found that it converged for $62 \mathrm{~L}$ (interestingly, when considering a periodic bulk two atom cell, we required a k-point mesh $31 \times 31 \times 31$, giving equivalent sampling).

Hybrid functional band structure For the hybrid functional calculations, owing to the large computational cost, the charge density was found self-consistently using a $4 \times 4 \times 1$ $\mathrm{k}$-point mesh.

To start, we tested the influence of slab thickness on three key properties: the $\mathrm{Si}$ dimer bond length and buckling angle; and the surface energy. The structural properties are relatively weakly dependent on the slab thickness (Table 1): the bond length and buckling angle both converge at $14 \mathrm{~L}$. Performing calculations with a slab of less than 14 $\mathrm{L}$ is probably acceptable for the study of surface geometry, but will require some care. On the other hand, surface energy convergence is only obtained when the slab thickness is greater than $22 \mathrm{~L}$, with the surface energy for a $14 \mathrm{~L}$ thick slab $114 \mathrm{meV}$ per dimer higher than the converged value. We recall the discussion of the relative stability of the $c(4 \times 2)$ and $p(2 \times 2)$ reconstructions [10, 18, 19, which involved energy differences of a few $\mathrm{meV}$, for which the error in surface energy, even at $14 \mathrm{~L}$, is far from negligible. For calculations considering reaction, adsorption, or diffusion at the $\mathrm{Si}(100)$ surface, slab thickness needs to be tested and chosen carefully.

We now turn to the key electronic structure: DOS and the band gap, on which the slab thickness has a profound effect. Figure 11(a) shows the DOS projected onto a Si dimer for slab thicknesses from $6 \mathrm{~L}$ to $78 \mathrm{~L}$. The common features among these DOS are two peaks in the filled states, and one broad and one pronounced peak in the empty states: these are often referred to as $\pi_{1}, \pi_{2}, \pi_{1}^{*}$ and $\pi_{2}^{*}$, as labeled in Fig. 1)(a) [12, 20]. Their appearance agrees well with scanning tunneling spectroscopy results (See 
(a)

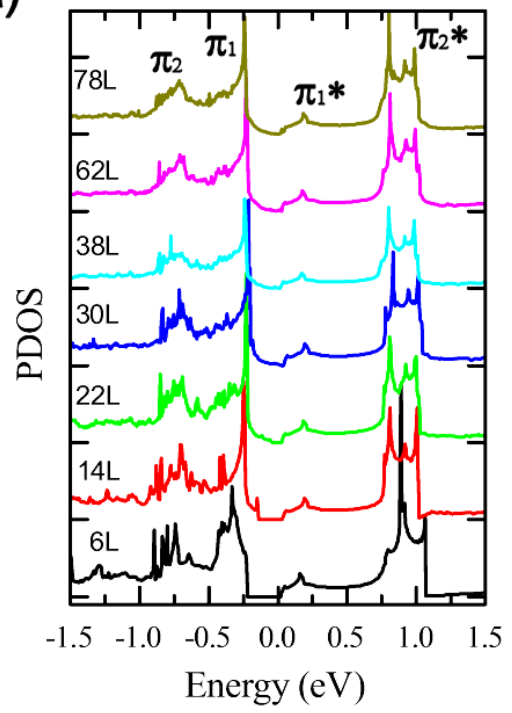

(b)

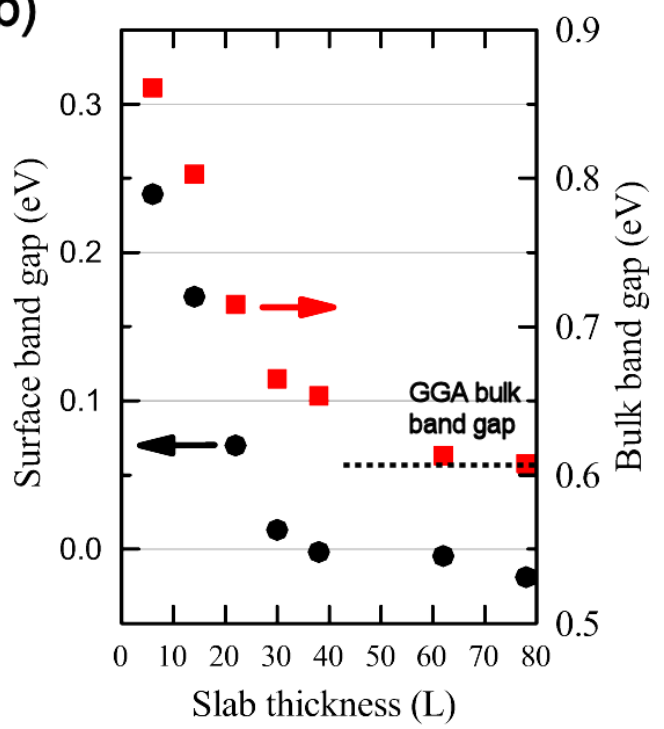

Figure 1. (Color online) (a) PDOS of Si dimer calculated with $\operatorname{Si}(100) p(2 \times 2)$ slabs. All energies are shifted so that the bottom of the potential of Si atoms averaged over the top five layer of each slab has the same value. (b) Surface (circle) and bulk (square) band gaps as a function of the slab thickness. The bulk band gap sizes were found from PDOS of the middle layer in each slab. The GGA bulk band gap value obtained by a converged bulk calculation is indicated by a broken line.

Fig. A1). The key effect of increasing slab thickness that can be seen is a change in the shape and character of the valence band maximum (VBM). As will become clear below, the VBM of thin slabs are dominated by the DB states, while those of thicker slabs are dominated by bulk states. To the best of our knowledge, the VBM character of a clean $\mathrm{Si}(100)$ surface has not been well studied yet, and we will discuss this based on the results of band calculations below.

The second major effect of the increasing slab thickness is a reduction of the surface band gap. Figure. 1(b) shows the surface and bulk band gaps (gap at the center of the slab) as a function of the slab thickness. The reduction of the surface band gap with slab thickness is strongly correlated with the reduction of the bulk band gap, and the development of the bulk bands. When the slab thickness is $38 \mathrm{~L}$ or greater, the bottom of the $\pi_{1}^{*}$ state overlaps with the top of the valence band, which has become a bulk state, and the surface band gap closes. It is maybe not surprising that there is some dependence of gap on slab thickness, as, a slab will behave as a form of quantum well, with a thin slab strongly confining its eigenstates, giving a larger band gap [21]. In our work, we calculated the fully converged bulk band gap of silicon to be $\sim 0.607 \mathrm{eV}$, which is only reached when a slab calculation with $62 \mathrm{~L}$ or more is performed.

In order to gain further insights into the influence of the slab thickness on the electronic structure, we have examined the band structure of the $\operatorname{Si}(100)-p(2 \times 2)$ surface with various slab thicknesses. Figure 2 shows valence band diagrams near the Fermi level along the dimer row direction $\left(\Gamma-\frac{1}{2} \mathrm{~J}^{\prime} \Gamma\right.$ line $)$. We plotted the partial charge densities 

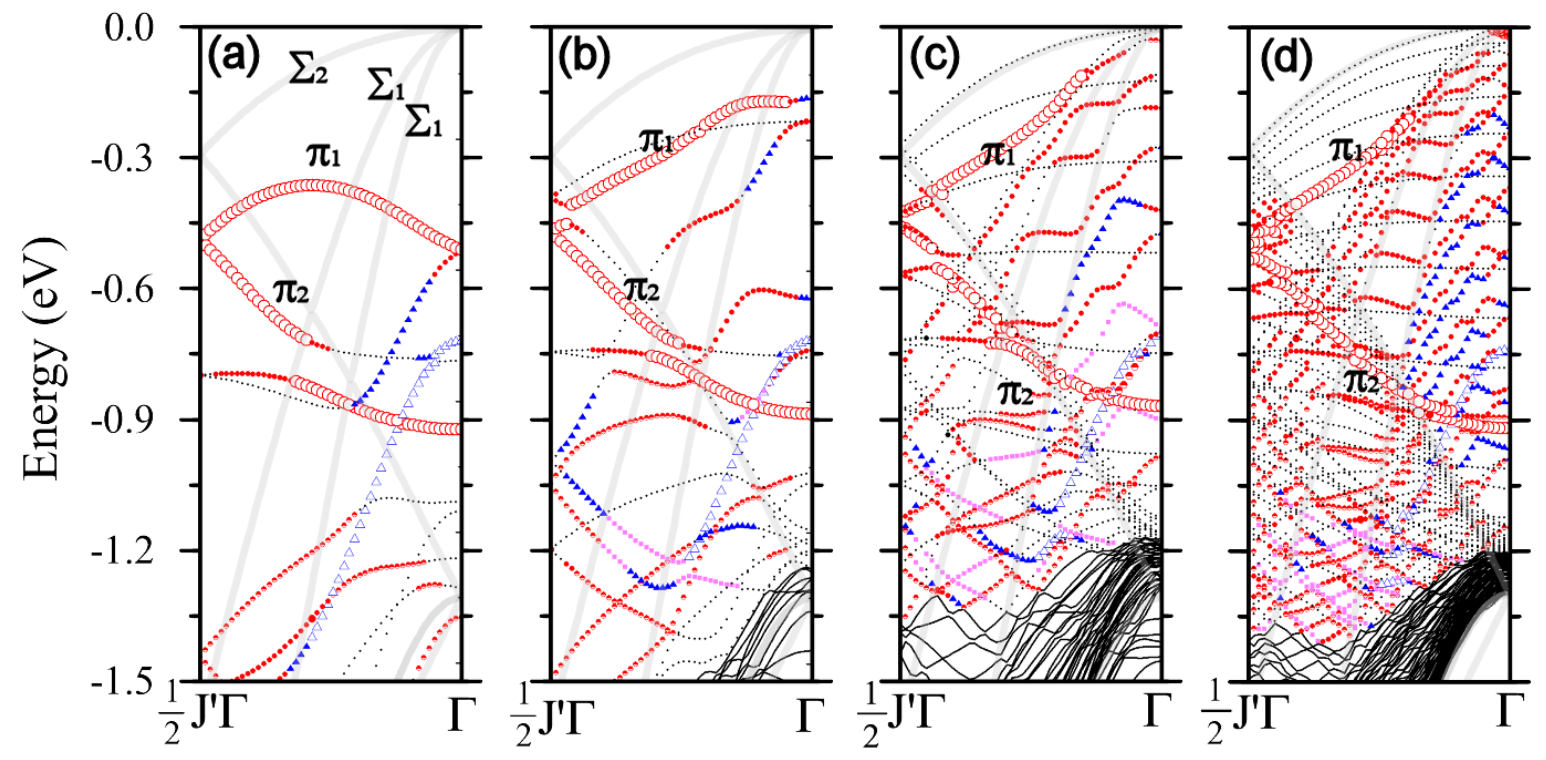

Figure 2. (Color online) Band structures for the $\operatorname{Si}(100) p(2 \times 2)$ surface along the dimer row direction $\left(\Gamma-\frac{1}{2} \mathrm{~J}^{\prime} \Gamma\right.$ line). Slab thickness used are (a) $6 \mathrm{~L}$, (b) $14 \mathrm{~L}$, (c) 38 $\mathrm{L}$, and (d) $78 \mathrm{~L}$. All bands are drawn with reference to the bottom of the potential of Si atoms averaged over the top five layer of each slab. Red open circles represent eigenstates with intense and localized charges on DB assigned by their charge values being larger than (a) 10, (b) 5.0, (c) 2.0, and (d) $1.0 \times 10^{-3} \mathrm{e} / \AA^{3}$; Red dots: hybrid states of DB and bulk states whose charge density is less than the foregoing criteria; Red half filled circles: hybrid states of $\mathrm{DB}, \mathrm{db}$ and bulk states; Blue open triangles: intense and localized BB state; Blue solid triangles: hybrid states of $\mathrm{BB}$ and bulk states; Pink squares: hybrid states of $\mathrm{BB}, \mathrm{db}$ and bulk states; Black dots: pure bulk states. Solid lines are unchecked bands. Gray lines are bulk bands (at $k_{z}=0$ ) obtained separately by a bulk calculation with a $p(1 \times 1) 4$ atom cell.

for each eigenstate at different k-points in the Brillouin zone (BZ) to assign the character (bulk, DB, back-bond) to the band as it moves through the BZ. Typical isosurfaces of the charge density for the $78 \mathrm{~L}$ slab are shown in Fig. 3 and in Fig. A2, The major surface states are the DB states $\pi_{1}$ and $\pi_{2}$ (red open circles). Both the $\pi_{1}$ and $\pi_{2}$ bands show intense charge densities on the Si dimer atoms and they decay within a few layers of the surface. The $\pi_{1}$ state bears charges localized on the up atom of the Si dimer, while the $\pi_{2}$ state has most of charges on the up atom and some extended onto the down atom as well.

We observe that the band shape and width of $\pi_{1}$ drastically changes with the increase of the slab thickness, while those of $\pi_{2}$ are only slightly changed by the slab thickness. The surface band maximum (SBM) appears half way along the $\Gamma-\frac{1}{2} \mathrm{~J}^{\prime} \Gamma$ line for $6 \mathrm{~L}$. Correspondingly, the $\pi_{1}$ band width is only $0.13 \mathrm{eV}$. With increasing thickness, the SBM moves toward the $\Gamma$ point and the band width also widens. Eventually, the SBM reaches to the $\Gamma$ point when the slab thickness is greater than $20 \mathrm{~L}$ and the band width converges, to approximately $0.40 \mathrm{eV}$, for thickness greater than $30 \mathrm{~L}$ (see Table A1).

The convergence of the surface states relies on more than just slab thickness: it 

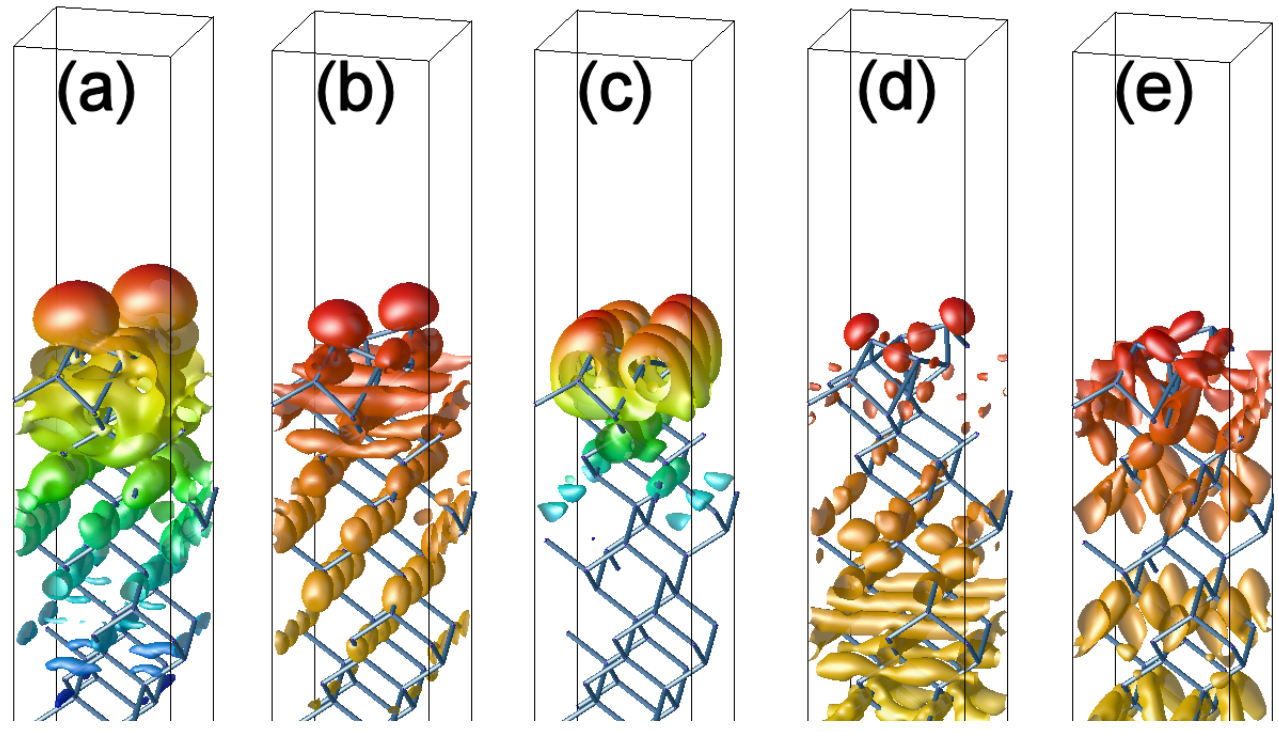

Figure 3. (Color online) Typical isosurface maps of charge density of the individual eigenstate calculated with a $\operatorname{Si}(100) p(2 \times 2)-78 \mathrm{~L}$ slab. (a) DB state $(\bigcirc)$, (b) $\mathrm{DB}+$ bulk hybrid state $(\bullet),(\mathrm{c}) \mathrm{BB}$ state $(\triangle),(\mathrm{d}) \mathrm{DB}+\mathrm{db}+$ bulk hybrid state $(\ominus)$, (e) $\mathrm{BB}+\mathrm{db}+$ bulk hybrid state $(\square)$. Isosurface value: $1.0 \times 10^{-3} \mathrm{e} / \AA^{-3}$. Color scale represents height in $\mathrm{z}$ direction. Each symbols are used in Fig. 2 to group types of states.

also requires precise reproduction of the bulk electronic structure in the slab. In Fig. 2 . the eigenstates with no charge density on the Si dimers (i.e. pure bulk bands) are plotted with black dots. For comparison, conventional bulk bands, calculated at $k_{z}=0$ with a repeated cell consisting of four Si atoms, are superimposed as thick gray curves and labeled as $\Sigma_{1}, \Sigma_{1^{\prime}}$ and $\Sigma_{2}$. As might be expected, the $6 \mathrm{~L}$ slab calculation does not produce any bulk bands near the surface bands [Fig. 2(a)]. With increasing slab thickness, the bulk bands develop, and the VBM becomes dominated by a bulk state, instead of the DB state, when the slab thickness is greater than 30 L. Full agreement between bulk valence bands in slab and bulk calculations is only obtained for a slab thickness of $60 \mathrm{~L}$ or more. This thickness matches with that of the slab calculation in which the bulk band gap agrees with the value of a repeated cell bulk calculation, as seen in Fig. 1 (b).

In a slab calculation, where the electronic states in the sample have no periodicity perpendicular to the surface and Bloch's theorem does not apply, extra bands appear and develop with increasing slab thickness (in contrast to a bulk calculation which requires increasing BZ sampling for convergence). The increase in number of bands and the development of their shape is seen clearly in Fig. 2. Some of the bands that appear with increasing thickness have charge density on both the Si dimers and the bulk atoms, as seen in Fig. 3 (b). These bands are hybridized between surface and bulk states. These hybridizations are prominent for the $\pi_{1}$ band, particularly near the $\Gamma$ point. As the slab thickness, and hence number of additional bands, becomes greater, more band mixing occurs: a thicker slab shows a larger number of hybridized bands. 
This causes the charge density of the $\pi_{1}$ band near the $\Gamma$ point to redistribute into the slab, resulting in an apparent absence of the $\pi_{1}$ band near the $\Gamma$ point, while the same band near the $\frac{1}{2} \mathrm{~J}^{\prime} \Gamma$ point, and the $\pi_{2}$ band across the BZ, are well localized at the surface regardless of slab thickness (See Fig. A3).

To investigate what causes this change in surface band energy and shape, we simplified the problem. We calculated bands for $2 \times 1$ symmetric (flat) and asymmetric (buckled) dimers with $6 \mathrm{~L}$ and $38 \mathrm{~L}$ slabs. The only effect that causes the surface band to change shape is hybridization with the bulk bands. The $\pi$ band of the symmetric dimer in Fig. 4(c) does not change its shape even though the slab thickness increases from $6 \mathrm{~L}$ to $38 \mathrm{~L}$ : since the $\pi$ band is situated in the bulk band gap, the hybridization is weak. On the other hand, the $\pi$ band of the asymmetric dimer changes its shape with slab thickness and this is not due to any structural change, but only due to the band mixing between the $\pi$ and the bulk bands. The increase in slab thickness develops the bulk bands and raises their energy predominantly around the $\Gamma$ point; simultaneously, the hybridization lifts the $\pi$ band's energy near the $\Gamma$ point as well.

These hybridizations are clearest between the $\Gamma$ point and a point halfway to the $\mathrm{J}^{\prime}$ point. This tendency is kept when the bands are folded and transformed in the surface Brillouin zone by switching the surface cell from $2 \times 1$ periodicity to the $p(2 \times 2)$. Correspondingly, the $\pi_{2}$ band, which originates from the second half of the $\pi$ band in the smaller cell, does not change in its shape with slab thickness. Hybridization between surface and bulk bands is inevitable in slab calculations, so a precise calculation of surface states will require the convergence of bulk states. It is interesting to note that hybridized bands associated with bulk states, back bond (BB) and dimer bond (db) are also found in Fig. 2 .

We have also examined the effect of slab thickness on the band structure along other high symmetry lines in the BZ. A comparison of band structures of $\mathrm{Si}(100)$ between $10 \mathrm{~L}$ and $38 \mathrm{~L}$ is displayed in Fig. 5. With a slab that is $10 \mathrm{~L}$ or thinner, as is typically used to model the Si(100) surface, the SBM tends to appear in the J- $\frac{1}{2} \mathrm{KJ}$ line [Fig. 5(a)]. This result can be found in previous reports [11, 10, 12, 22]. However, the SBM is actually located at the $\Gamma$ point in converged band structures calculated with a sufficiently thick slab [Fig. 5(b)]. This agrees with the observations of direct transition of the surface band gap [23, 24].

Due to the narrowing of the bulk band gap with the slab thickness, the bottom of the unoccupied DB band $\left(\pi_{1}^{*}\right)$ overlaps the top of the valence band when the slab thickness is greater than 30 layers. It might be that this overlap affects the development of the bands, so to check this, we carried out a band calculation for the $38 \mathrm{~L}$ slab with a screened hybrid exchange-correlation functional (HSE06) [25, 26, 27]. These functionals generally alleviate the standard DFT problem with band gaps that are too small. The results are shown in Fig. 5(c), where it is clear that the band gap has opened. There are no other differences in the band structure between the conventional and hybrid functional calculations. Our discussions, based on standard DFT, are therefore valid and unaffected by the band gap. 

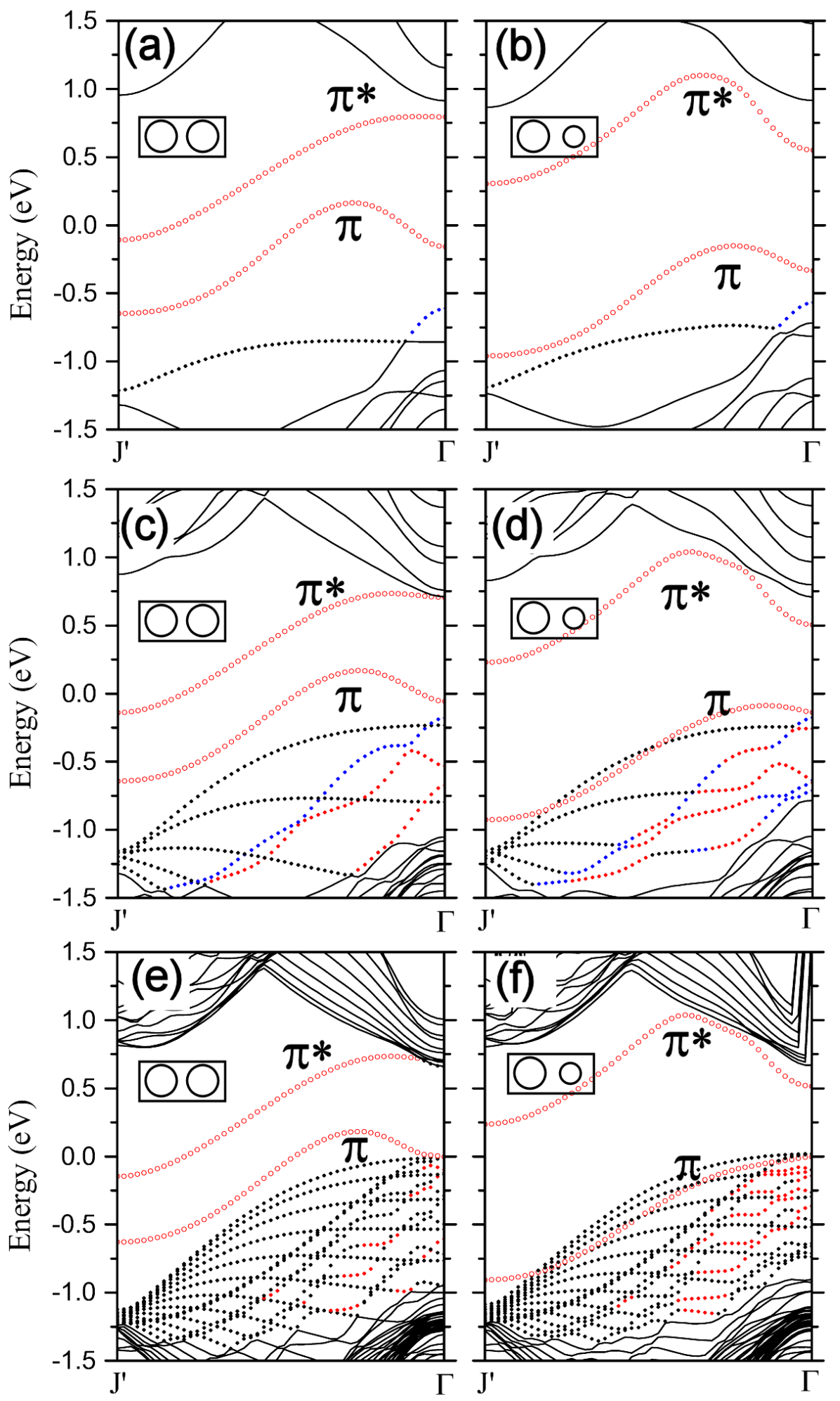

Figure 4. Band structures of (a), (c), (e) $2 \times 1$ symmetric dimer and (b), (d), (f) $2 \times 1$ asymmetric dimer surfaces obtained with slabs of: (a), (b) 6L; (c), (d) 14L; (e), (f) 38L. Red open circle: DB states. red dots: hybrid states of DB and bulk states, black dots: bulk states, solid line: unchecked bands. 
(a)

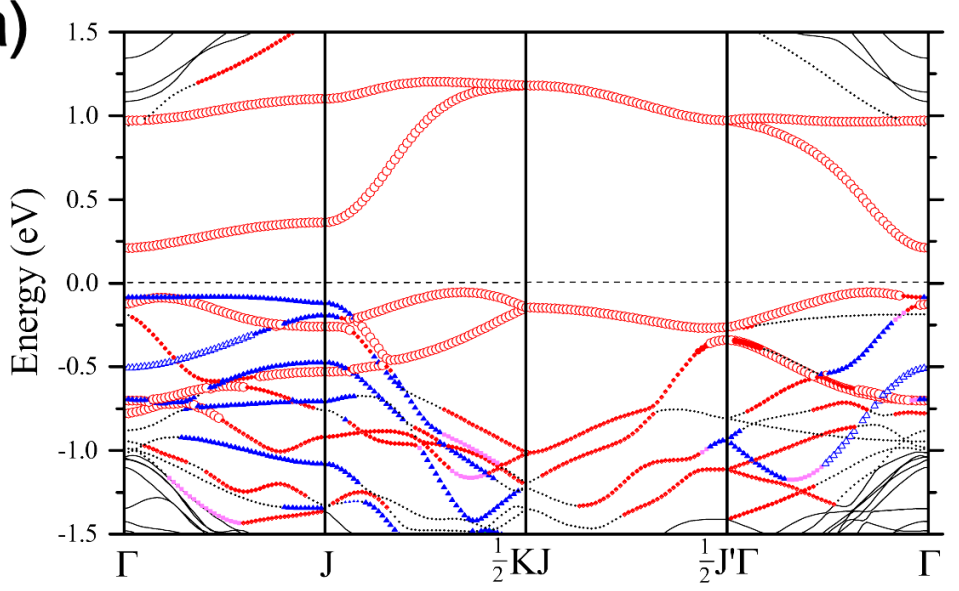

(b)

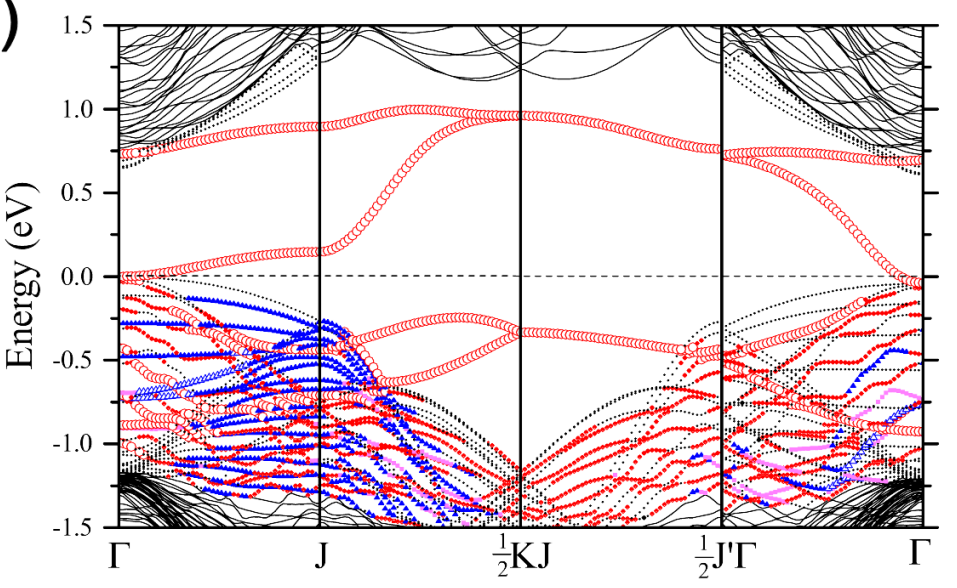

(c)

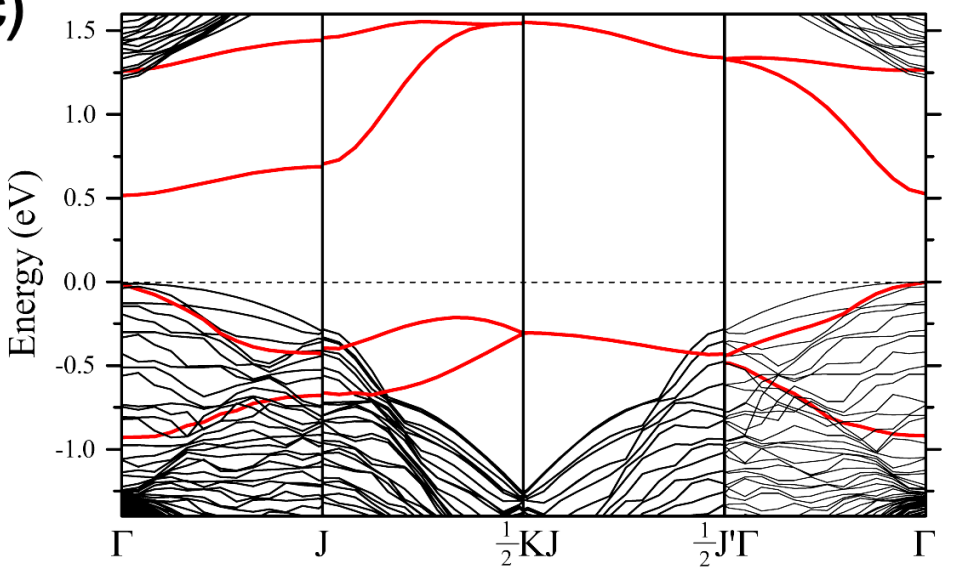

Figure 5. (Color online) Band structures of $\operatorname{Si}(100) p(2 \times 2)$ calculated for slab thicknesses of (a) $10 \mathrm{~L}$, (b) and (c) $38 \mathrm{~L}$. (a) and (b) were calculated by GGA PBE functional and (c) by hybrid exchange-correlation functional (HSE06). To obtain the hybrid functional bands, both structural and electronic relaxations were also done by using HSE06. The usage of symbols are the same as that in FIG. 2. The DB states represented with red open circle are drawn in red lines in (c). 


\section{Conclusion}

We have shown that DFT calculations for the electronic structure of the $\mathrm{Si}(100)$ surface require a much thicker slab than is conventionally used. A study for simple geometrical structures will be adequate with a thin slab of around 15 layers, though 20-25 layers are required for convergence of the surface energy. Treatment of the precise surface electronic structure needs a slab of at least 30 layers, while the full convergence of both

surface and bulk electronic structure requires a slab of 60 layers or more. It is important to determine which properties need to be calculated and choose an appropriate slab size. With advances in experimental techniques, the desired accuracy from DFT modeling has reached an energy resolution in the meV range, suggesting that calculations should be performed with a significantly thicker slab than is the norm.

\section{Acknowledgments}

This work was in part supported by MEXT KAKENHI (24510160).

\section{References}

[1] Veldhorst M, Yang C H, Hwang J C C, et al. 2015 Nature 526410

[2] Radny M W, Shah G A, Schofield S R, Smith P V and Curson N J 2008 Phys. Rev. Lett. 100 246807

[3] Yan B, Yam C, da Rosa A L and Frauenheim T 2009 Phys. Rev. Lett. 103189701

[4] Radny M W, Shah G A, Schofield S R, Smith P V and Curson N J 2009 Phys. Rev. Lett. 103 189702

[5] Shah G A, Radny M W, Smith P V and Scofield S R 2012 J. Phys. Chem C 1166615

[6] Smeu M, Guo H, Ji W and Wolkow R A 2012 Phys. Rev. B 85195315

[7] Vazhappilly T and Micha D A 2014 J. Phys. Chem. C 1184429

[8] Martinez A, Kalna K, Sushko P V, Shluger A L, Barker J R and Asenov A 2009 IEEE Trans. Nanotechnol. 8159

[9] Seo H, Hatch R C, Ponath P, Choi M, Posadas A B and Demkov A A 2014 Phys. Rev. B 89115318

[10] Fritsch J and Pavone P 1995 Surf. Sci. 344159

[11] Zhu Z, Shima N and Tsukada M 1989 Phys. Rev. B 4011868

[12] Ramstad A, Brocks G and Kelly P J 1995 Phys. Rev. B 5114504

[13] Takayama A private communication

[14] Kresse G and Hafner J 1993 Phys. Rev. B 47558

[15] Kresse G and Furthmuller J 1996 Phys. Rev. B 5411169

[16] Perdew J P, Burke K and Ernzerhof M 1996 Phys. Rev. Lett. 773865

[17] Blöchl P E, Jepsen O and Andersen O K 1994 Phys. Rev. B 4916223

[18] Inoue K, Morikawa Y, Terakura K and Nakayama M 1994 Phys. Rev. B 4914774

[19] Nakamura J and Natori A 2005 Phys. Rev. B 71113303

[20] Okada H, Fujimoto Y, Endo K, Hirose K and Mori Y 2001 Phys. Rev. B 63195324

[21] Delley B and Steigmeier E F 1995 Appl. Phys. Lett. 672370

[22] Zhou M, Liu Z, Wang Z, Bai Z, Feng Y, Lagally M G and Liu F, Phys. Rev. Lett. 111246801

[23] Goldmann A, Koke P, Mönch W, Wolfgarten G and Pollmann J 1986 Surf. Sci. 169438

[24] Enta Y, Kinoshita T, Suzuki S and Kono S 1987 Phys. Rev. B 369801

[25] Heyd J, Scuseria G E and Ernzerhof M 2003 J. Chem. Phys. 1188207

[26] Heyd J and Scuseria G E 2004 J. Chem. Phys. 1211187 


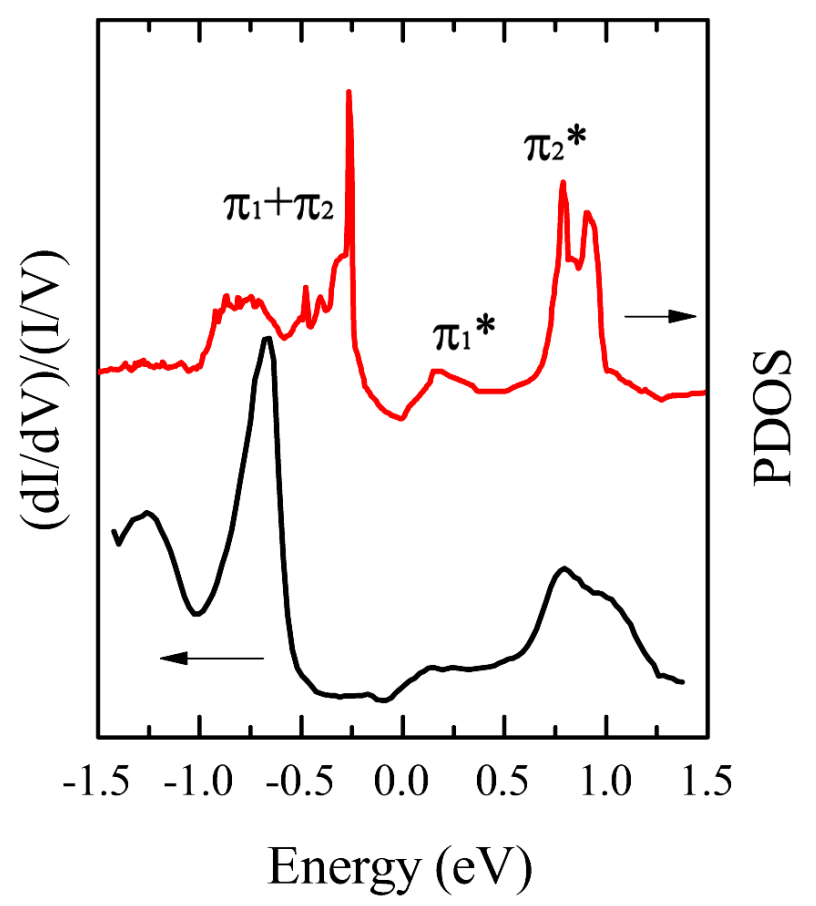

Figure A1. Tunneling spectrum and DFT-PDOS of Si dimer on the $\mathrm{Si}(100)$ surface. Tunneling spectrum was obtained at $78 \mathrm{~K}$ with a setpoint of $\mathrm{Vs}=+1.5$ and $\mathrm{I}=3.5 \mathrm{nA}$. PDOS was obtained with a $62 \mathrm{~L}$ slab.

[27] Heyd J, Scuseria G E and Ernzerhof M 2006 J. Chem. Phys. 124219906

\section{Appendix A. Extra Figures and Tables}

The table and figures below are not central to our study, but add extra detail in some areas, and are included for completeness and information.

Table A1. Band width of $\pi_{1}$ and $\pi_{2}$ bands in the $\Gamma-\frac{1}{2} \mathrm{~J}^{\prime} \Gamma$ line.

Slab thickness (L) Bandwidth (eV)

\begin{tabular}{ccc} 
& $\pi_{1}(\mathrm{eV})$ & $\pi_{2}(\mathrm{eV})$ \\
\hline 6 & 0.130 & 0.434 \\
14 & 0.312 & 0.411 \\
22 & 0.385 & 0.434 \\
30 & 0.399 & 0.426 \\
38 & 0.399 & 0.416 \\
62 & 0.400 & 0.405 \\
78 & 0.410 & 0.405
\end{tabular}



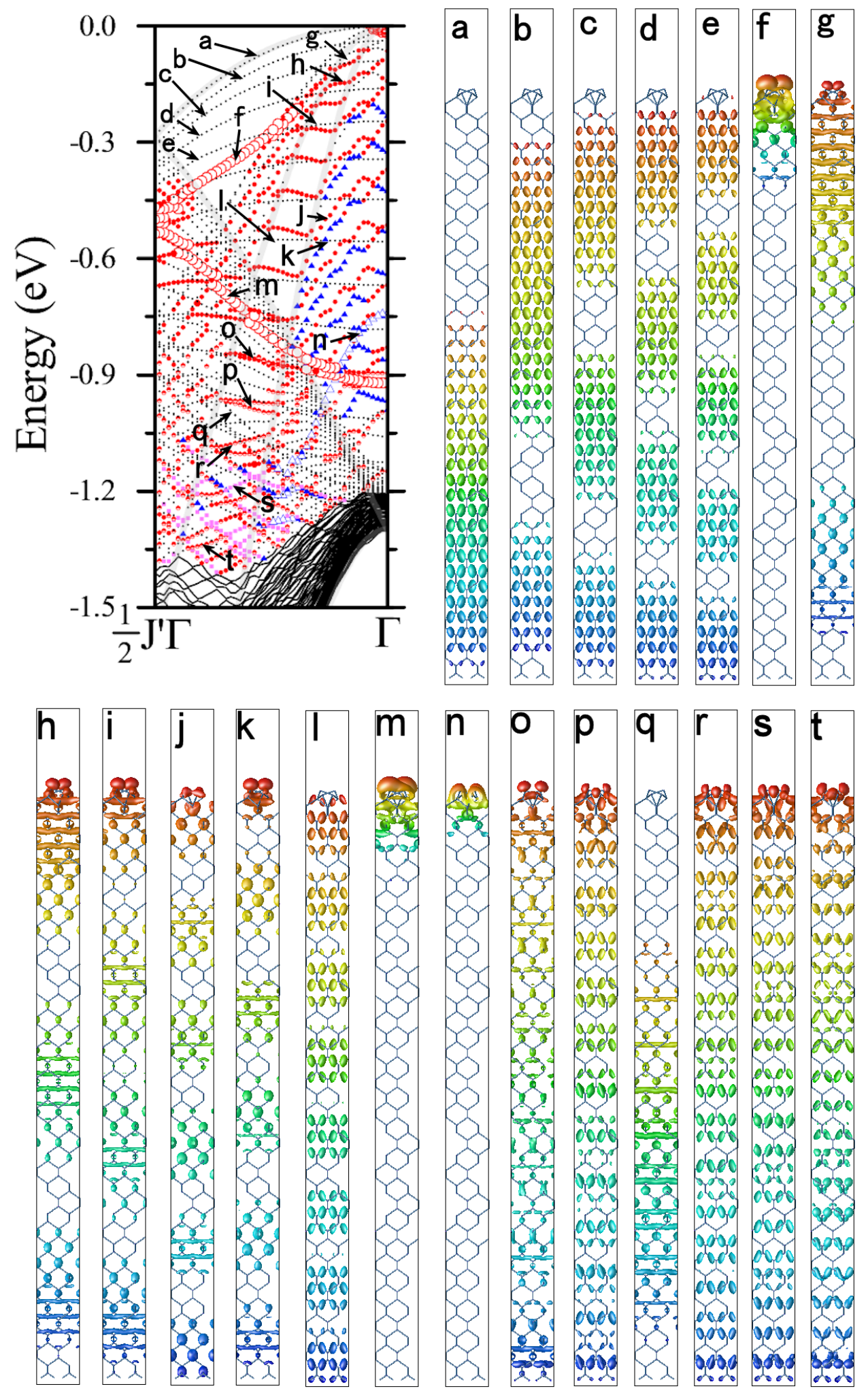

Figure A2. Isosurface charge density distributions of eigenstates indicated by labels in the band diagram of $\operatorname{Si}(100)-p(2 \times 2)-78 \mathrm{~L}$. Isosurface value is $1 \times 10^{-3} \mathrm{e} / \AA^{3}$. 
Importance of bulk states for the electronic structure of semiconductor surfaces

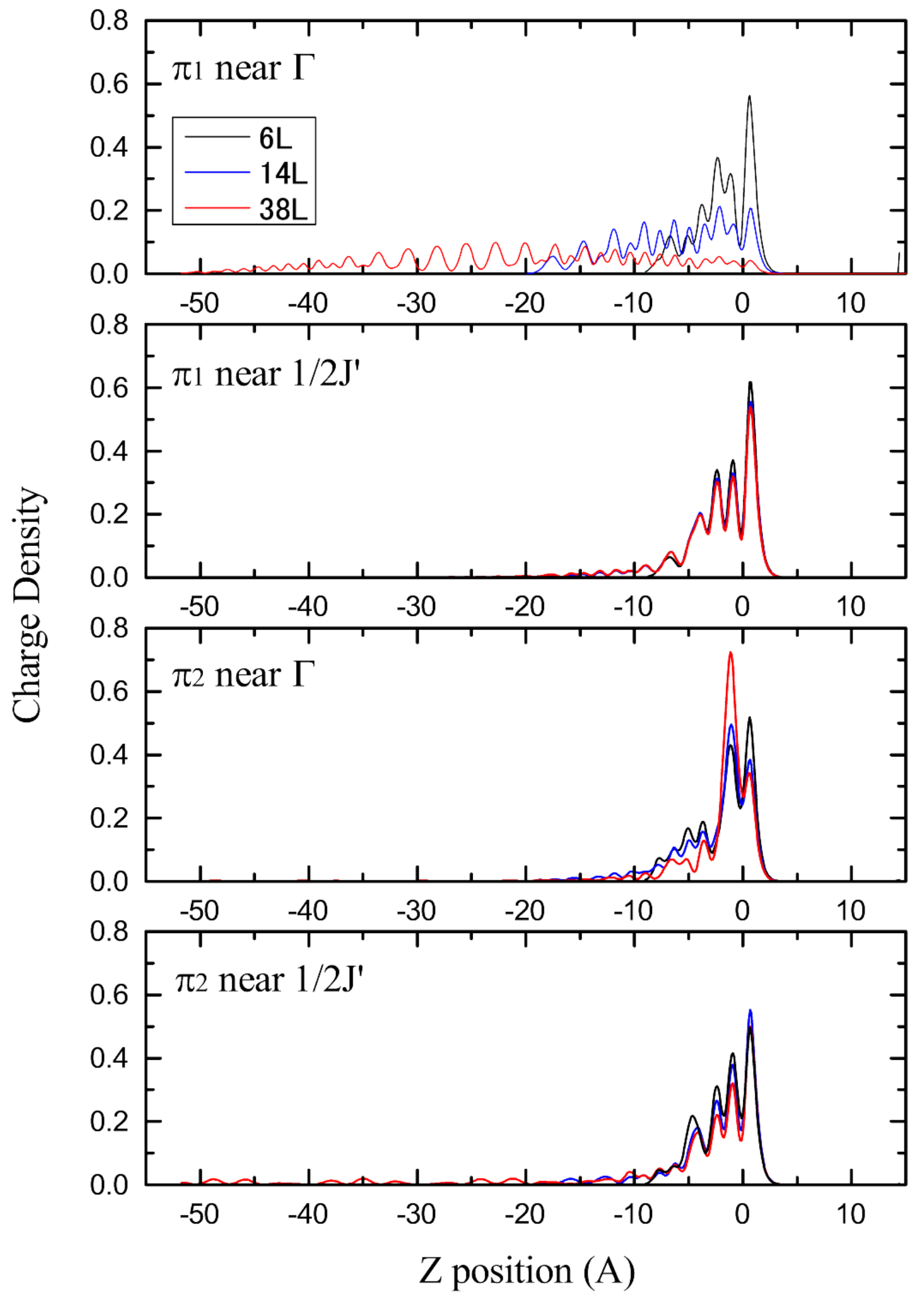

Figure A3. Charge density of $\pi_{1}$ and $\pi_{2}$ states near $\Gamma$ point and $1 / 2 \mathrm{~J}$ ' as a function of $\mathrm{Z}$ position for 6,14 , and $38 \mathrm{~L}$ slabs. 